DAMTP

University of Cambridge

DAMTP-1998-143

UTAP-308

RESCEU-55/98

May 11, 2018

\title{
Inverse Cascade of Primordial Magnetic Field in MHD Turbulence
}

\author{
Tetsuya Shiromizu 由 \\ DAMTP, University of Cambridge \\ Silver Street, Cambridge CB3 9EW, UK \\ Department of Physics, The University of Tokyo, Tokyo 113-0033, Japan \\ and \\ Research Centre for the Early Universe(RESCEU), \\ The University of Tokyo, Tokyo 113-0033, Japan \\ to be published in Physics Letter B
}

\begin{abstract}
The feature of the spectrum of primordial magnetic field is studied by using renormalization group analysis in magnetohydrodynamics. Taking account of the renormalized resistivity at the fixed point, we show that the scaling of the typical scale with time obeys $L(t) \sim t^{2 / 5}$ for random initial condition.
\end{abstract}

The magnetic fields observed in the various astrophysical scales [1] is one of the important problems in cosmology[2]. The generation mechanism of magnetic field in proto-galaxy scale was already proposed by Kulsrud et al[3]. However, as this mechanism cannot explain the magnetic fields in intergalactic mediums and interclusters regions where matter is poor, it seems that the problem of the origin of magnetic fields still remains. This origin is

\footnotetext{
${ }^{1}$ JSPS Postdoctal Fellowship for Research Abroad
} 
often attributed to the very early universe. Some attractive mechanisms in the course of cosmological phase transitions have been proposed [4]. In these mechanisms, the coherent length of the generated magnetic field is much smaller than the galaxy scale etc., because the magnetic field is generated by micro physical processes. Thus, one needs to investigate the process in which the small magnetic domains evolve to larger scales observed in the present universe. Since Kulsrud and Anderson[5] pointed out that the kinetic dynamo theory [6] breaks down in interstellar mediums one cannot expect enough amplification of the magnetic field. Hence, we must re-analyze the evolution of MHD (Magnetohydrodynamics) from the starting point.

Recently, Brandenburg et al[7] showed numerically that the inverse cascade occurs by using cascade model, and the occurrence of inverse cascade is also showed analytically by Olesen [9]. In this paper, we reconsider Olesen's study from the viewpoint of the renormalization group. We show that the case considered in Ref. [9] corresponds to our analysis at the fixed point. Then we discuss the evolution of the coherent length near the fixed point.

Almost all of astrophysical systems have high magnetic Reynolds number because it is highly conductive in the typical astrophysical scales. Thus, MHD turbulence will appear in such system with fluid flow and we can use the statics of the turbulence to analize this system. We will apply the result obtained in Ref. 110 to the evolution of primordial magnetic field in our study.

First of all, we review the renormalization group analysis of MHD. The basic equations of the incompressible MHD are given by

$$
\partial_{t} \mathbf{P}+\mathbf{Q} \cdot \nabla \mathbf{P}=-\nabla p_{*}+\gamma_{+} \Delta \mathbf{P}+\gamma_{-} \Delta \mathbf{Q}+\mathbf{f},
$$

and

$$
\partial_{t} \mathbf{Q}+\mathbf{P} \cdot \nabla \mathbf{Q}=-\nabla p_{*}+\gamma_{+} \Delta \mathbf{Q}+\gamma_{-} \Delta \mathbf{P}+\mathbf{g}
$$

where $\mathbf{P}=\mathbf{v}+\mathbf{B}, \mathbf{Q}=\mathbf{v}-\mathbf{B}, p_{*}=p+(1 / 2) \mathbf{B}^{2}$, and $\gamma_{ \pm}=\nu \pm \eta$. The $\mathbf{f}$ and $\mathbf{g}$ are the stirring forces and they are assumed to satisfy the statistical correlation,

$$
\begin{gathered}
\left\langle f_{i}(k) f_{j}(q)\right\rangle=2 k^{-y} A_{0}(2 \pi)^{4} J_{i j}(\mathbf{k}) \delta^{4}(k+q) \\
\left\langle f_{i}(k) g_{j}(q)\right\rangle=2 k^{-y} B_{0}(2 \pi)^{4} J_{i j}(\mathbf{k}) \delta^{4}(k+q),
\end{gathered}
$$


and

$$
\left\langle g_{i}(k) g_{j}(q)\right\rangle=2 k^{-y} A_{0}(2 \pi)^{4} J_{i j}(\mathbf{k}) \delta^{4}(k+q),
$$

where $J_{i j}(\mathbf{k})=\delta_{i j}-k_{i} k_{j} /\left|\mathbf{k}^{2}\right|$ and $A_{0}, B_{0}$ are constants. The power of $k$ should be decided by the initial condition. These forces are introduced so that the above system is equivalent to the original system in the inertial range(correspondence principle[1]). The solution is written as

$$
\hat{G}_{0}^{-1} \hat{P}_{i}(k)=\hat{f}_{i}(k)-i J_{i j k}(\mathbf{k}) \int d q\left(\begin{array}{c}
Q_{j}(k-q) P_{k}(q) \\
P_{j}(k-q) Q_{k}(q)
\end{array}\right),
$$

where $\hat{P}_{i}(k)=\left(\begin{array}{c}P_{i}(k) \\ Q_{i}(k)\end{array}\right), \hat{f}_{i}(k)=\left(\begin{array}{c}f_{i}(k) \\ g_{i}(k)\end{array}\right)$ and $J_{i j k}(\mathbf{k})=k_{j} J_{i k}(\mathbf{k}) . \hat{G}_{0}(k)$ is the bare Green function written as

$$
\hat{G}_{0}^{-1}(k)=\left(\begin{array}{cc}
-i \omega+\gamma_{+}|\mathbf{k}|^{2} & \gamma_{-}|\mathbf{k}|^{2} \\
\gamma_{-}|\mathbf{k}|^{2} & -i \omega+\gamma_{+}|\mathbf{k}|^{2}
\end{array}\right),
$$

Now, we divide the $k$-space into two parts, that is, large scale modes $0<k<\Lambda e^{-r}$ and small scale modes $\Lambda e^{-r}<k<\Lambda$, where $\Lambda$ is the ultraviolet cut off. Then, we construct the effective equation for the large scale mode by integrating out the small scale modes. The result becomes

$$
\hat{G}^{<-1}(k ; r) \hat{P}_{i}^{<}(k)=\hat{f}_{i}^{<}(k)-i \lambda_{0} J_{i j k}(\mathbf{k}) \int d q\left(\begin{array}{c}
Q_{j}^{<}(k-q) P_{k}^{<}(q) \\
P_{j}^{<}(k-q) Q_{k}^{<}(q)
\end{array}\right),
$$

where subscript $<$ means $k \in\left[0, \Lambda e^{-r}\right]$, and $\hat{G}(k ; r)$ is the dressed Green function. $\lambda_{0}$ is the expansion parameter introduced technically. Now, $\gamma_{ \pm}$depends on the renormalization parameter, $r$, and obey the following equation,

$$
\frac{d \gamma_{ \pm}(r)}{d r}=\frac{\lambda_{0}^{2}}{4} A_{d} A_{0} \frac{\gamma_{+}^{2}(r)}{\gamma_{-}^{2}(r)} \frac{1}{\nu^{2}(r) \eta^{2}(r)} \frac{e^{\epsilon r}}{\Lambda^{\epsilon}} F_{ \pm}\left(\gamma_{+}, \gamma_{-}\right)
$$

where $A_{d}=1 /\left(120 \pi^{3 / 2}\right), \epsilon=1+y$, and $F_{ \pm}$are the functions of $\gamma_{ \pm}$, whose explicit expressions are not given here. From the above equation, one finds that the solution at the fixed point is $\gamma_{ \pm} \propto e^{\epsilon r / 3}$ [10].

Compared the eq. (6) with eq. (7), one can see easily that the system is invariant under the following scale transformation,

$$
\mathbf{k} \rightarrow \tilde{\mathbf{k}}=\mathbf{k} e^{r}=: \mathbf{k} \ell \quad \omega \rightarrow \tilde{\omega}=\omega \ell^{\alpha}
$$




$$
\begin{aligned}
& \mathbf{B}\left(\omega \ell^{\alpha}, \mathbf{k} \ell\right) \rightarrow \tilde{\mathbf{B}}(\tilde{\mathbf{k}}, \tilde{\omega})=\mathbf{B}(\mathbf{k}, \omega) \ell^{-\beta} \quad \mathbf{v}\left(\omega \ell^{\alpha}, \mathbf{k} \ell\right) \rightarrow \tilde{\mathbf{v}}(\tilde{\mathbf{k}}, \tilde{\omega})=\mathbf{v}(\mathbf{k}, \omega) \ell^{-\beta}(11) \\
& \nu(\ell) \rightarrow \tilde{\nu}=\nu(\ell) \ell^{\alpha-2} \quad \eta(\ell) \rightarrow \tilde{\eta}=\eta(\ell) \ell^{\alpha-2} \\
& \mathbf{f}(\mathbf{k}, \omega) \rightarrow \tilde{f}(\tilde{\mathbf{k}}, \tilde{\omega})=\mathbf{f}(\mathbf{k}, \omega) \ell^{\alpha-\beta} \quad \mathbf{g}(\mathbf{k}, \omega) \rightarrow \tilde{g}(\tilde{\mathbf{k}}, \tilde{\omega})=\mathbf{g}(\mathbf{k}, \omega) \ell^{\alpha-\beta} .
\end{aligned}
$$

From the requirement for the invariance of the stirring forces, one can obtain the relation,

$$
2 \beta=3 \alpha+(y+3)
$$

Now we can consider the evolution of the power spectrum $E(k, t)$,

$$
\left\langle B^{2}\right\rangle=\int d k E(k, t)
$$

From the scaling of $(10) \sim(13)$, the equation

$$
\ell^{5+2 \alpha-2 \beta} E(k, t)=E\left(\ell k, \ell^{-\alpha} t\right)
$$

holds. Defining the function $\psi(k, t)=k^{-5-2 \alpha-2 \beta} E(k, t)$, the above equation becomes

$$
\psi\left(\ell k, \ell^{-\alpha} t\right)=\psi(k, t)
$$

The solution is written as

$$
\psi(k, t)=\psi\left(k^{\alpha} t\right)
$$

Thus, the spectrum is given by

$$
E(k, t)=k^{2-\alpha-y} \psi\left(k^{\alpha} t\right)
$$

As $\alpha=\frac{5-y}{3}$ holds at the fixed point [10], we obtain

$$
E(k, t)=k^{(1-2 y) / 3} \psi\left(k^{(5-y) / 3} t\right)
$$

at the fixed point. The above expression is the same as one obtained by Olesen[9]. Furthermore, as $\partial_{t} E(k, t) \sim E(k, t) / t \sim \eta(k) k^{2} E(k, t)$ approximately at the fixed point, where $\eta(k) \propto k^{-\epsilon / 3}[10$, the time evolution of the typical length obeys

$$
L(t) \sim k^{-1} \propto t^{3 /(5-y)}
$$


Let us consider the case where the initial spectrum is white noise, $y=$ $-5 / 2$, that is, $E(k, 0)=k^{2} \psi(0)$. In this case, the typical correlation length becomes $L(t) \propto t^{2 / 5}$. If we assume that the magnetic field is generated in a cosmological phase transition at the temperature $T_{f}$ (the cosmic time $t_{f}$ ) and the coherent scale is the same as the bubble size, the present physical coherent scale is given by

$$
\begin{aligned}
L^{\mathrm{wn}}\left(t_{0}\right) & =L^{\mathrm{wn}}\left(t_{f}\right) \frac{a\left(t_{0}\right)}{a\left(t_{f}\right)}\left(\frac{t_{0}}{t_{f}}\right)^{2 / 5} \\
& \simeq 10^{35}\left(\frac{f_{b}}{10^{-4}}\right)\left(\frac{T_{f}}{100 \mathrm{GeV}}\right)^{-1 / 5} \mathrm{GeV}^{-1} \simeq 10^{-2} \times 10 \mathrm{Mpc},
\end{aligned}
$$

where $10 \mathrm{Mpc}$ is the Silk damping scale of the magnetic field[12] where the magnetic field with smaller scale is damped by the photon diffusion around the recombination epoch. $f_{b}$ is the ratio of the bubble size $L\left(t_{f}\right)$ to the horizon scale $H_{f}^{-1}$. For the electroweak phase transition, these typical values becomes $f_{b} \sim 10^{-4}$ and $T_{f} \sim 100 \mathrm{GeV}$ [13. This coherent length is $10^{12}$ times as much as the comovingly developed scale; $\sim 10 \mathrm{AU}$. We note that this result differs from one obtained by numerical simulations in cascade model, $L(t) \propto t^{0.25}[7]$. However, since we used the renormalized resistivity which contains the non-linear effect fully, our estimation might be more useful.

Next, to compare with the above result, we consider the case of Kraichnan spectrum $E(k, 0) \propto k^{-3 / 2}$, that is, $L(t) \propto t^{4 / 3}$, which is the one expected by the naive argument of Alfvén effect in the inertial range[14]. In this case, the naive estimation shows that the coherent scale exceeds the present horizon scale as follows,

$$
\begin{aligned}
L^{\mathrm{kra}}\left(t_{0}\right) & \simeq 10^{63}\left(\frac{f_{b}}{10^{-4}}\right)\left(\frac{T_{f}}{100 \mathrm{GeV}}\right)^{8 / 3} \mathrm{GeV}^{-1} \\
& \simeq 10^{28} \times 10 \mathrm{Mpc} \gg H_{0}^{-1} \sim 3000 \mathrm{Mpc}
\end{aligned}
$$

In this estimation, we need to study in the frame work of general relativity.

From the above investigation, the correlation length can become enough size to explain the macroscopic scale. Although the Silk damping effect erases the structure smaller than the Silk scale, there is no drastic change of the quantitative result after taking account of the reasonable viscosity term 8]. Finally, we note that these results does not depend on the helicity of the magnetic field. 


\section{Acknowledgements}

The author would like to thank Masahiro Morikawa and Ryoichi Nishi for their comments. The author is grateful to Gary Gibbons and DAMTP relativity group for their hospitality. He also thanks T. Uesugi for a careful reading of the manuscript of this paper. This work is supported by JSPS fellow.

\section{References}

[1] P.P. Kronberg, Pep. Prog. Phys. 57, 325(1994)

[2] For example, A. V. Olinto, 'Cosmological Magnetic Field' in the Proceeding of 3rd RESCEU Symposium 'Particle Cosmology', eds. K. Sato, T. Yanagida and T. Shiromizu, Univ. Acad. Press, p151(1998);

K. Enqvist, astro-ph/9803196

[3] R. M. Kulsrud, R. Cen, J. P. Ostriker and D. Ryu, Astrophys. J. 480(1997),481;

B. D. G. Chandran, Astrophys. J. 482(1997),156;

B. D. G. Chandran and O. Rodriguez, Astrophys. J. 490(1997), 156;

B. D. G. Chandran, Astrophys. J. 492(1998), 179

[4] B. Cheng and A. V. Olinto, Phys. Rev. D50(1994),2421;

G. Baym, D. Bödeker and L. McLerran, Phys. Rev. D53(1996), 662;

G. Sigl, A. V. Olinto and K. Jedamik, Phys. Rev. D55(1997), 4582;

T. Shiromizu, Phys. Rev. D58(1998), 107301

[5] R. M. Kulsrud and S. W. Anderson, Astrophys. J. 396(1992)606

[6] For example, E. N. Parker, Cosmological Magnetic Field (Oxford Univ. Press, Oxford, 1979)

[7] A. Brandenburg, K. Enqvist and P. Olesen, Phys. Rev. D54(1996)1291

[8] A. Brandenburg, K. Enqvist and P. Olesen, Phys. Lett. B391(1997)395

[9] P. Olesen, Phys. Lett. B398(1997)321 
[10] S. J. Camargo and H. Tasso, Phys. Fluids B4(1992)1199

[11] V. Yakhot and S. A. Orszag, J. Sci. Computing, 1(1986),3;

W. P. Dannevik, V. Yakhot and S. A. Orszag, Phys. Fluids 30(1987), 2021

[12] K. Jedamzik, V. Katalinic and A. V. Olinto, Phys. Rev. D57 (1998), 3264

[13] A. F. Heckler, Phys. Rev. D51(1995), 405

[14] D. Biskamp, Nonlinear Magnetohydrodynamics (Cambridge Univ. Press, 1993) 